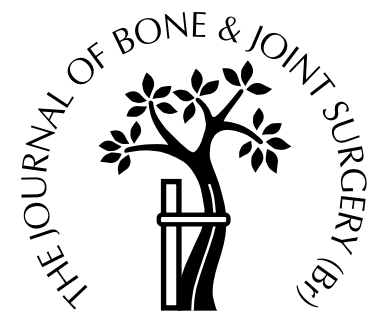

\title{
The appearance on MRI of vertebrae in acute compression of the spinal cord due to metastases
}

\author{
F. M. Khaw, S. A. Worthy, M. J. Gibson, A. Gholkar \\ From Newcastle General Hospital, Newcastle upon Tyne, England
}

$\mathbf{W}$ e studied MR images of the spine in a consecutive series of 100 patients with acute compression of the spinal cord due to metastases. All patients had documented neurological deficit and histologically proven carcinoma. MRI was used to localise bony metastatic involvement and soft-tissue impingement of the cord. A systematic method of documenting metastatic involvement is described.

A total of 43 patients had compression at multiple levels; 160 vertebral levels were studied. In 120 vertebrae $(\mathbf{7 5 \%})$, anterior, lateral and posterior bony elements were involved. Soft-tissue impingement of the spinal cord often involved more than one quadrant of its circumference. In 69 vertebrae $(43 \%)$ there was concomitant anterior and posterior compression. Isolated involvement of a vertebral body was observed in only six vertebrae $\mathbf{( 3 . 8 \% )}$.

We have shown that in most cases of acute compression of the spinal cord due to metastases there is coexisting involvement of both anterior and posterior structures.

J Bone Joint Surg [Br] 1999;81-B:830-4.

Received 29 October 1998; Accepted after revision 8 February 1999

Compression of the spinal cord is one of the clearest indications for surgery in patients with metastatic vertebral disease. The surgical management depends on a knowledge

F. M. Khaw, FRCS, Lecturer in Orthopaedic Surgery

Department of Trauma and Orthopaedic Surgery, University of Newcastle upon Tyne, The Medical School, Framlington Place, Newcastle upon Tyne NE2 4HH, UK.

S. A. Worthy, FRCR, Consultant Radiologist

Department of Radiology, Royal Victoria Infirmary, Queen Victoria Road, Newcastle upon Tyne NE1 4LP, UK.

M. J. Gibson, FRCS, Consultant Orthopaedic Surgeon

Department of Orthopaedic Surgery, Freeman Hospital, Freeman Road, High Heaton, Newcastle upon Tyne NE7 7DN, UK.

A. Gholkar, FRCR, Consultant Neuroradiologist

Department of Neuroradiology, Newcastle General Hospital, Westgate

Road, Newcastle upon Tyne NE4 6BE, UK.

Correspondence should be sent to Mr F. M. Khaw.

(C)1999 British Editorial Society of Bone and Joint Surgery 0301-620X/99/59600 \$2.00 of the level and site of the compressive lesion, and the extent of bony metastatic involvement.

MRI is the investigation of choice in the evaluation of compression of the spinal cord due to metastases. ${ }^{1-3}$ It can identify the level(s) of involvement, the sites and the direction of compression.

While it is accepted that metastatic involvement of the vertebral body is common, there is no reported systematic documentation of the involvement of posterior structures. Our aim in this study was to evaluate systematically the pattern of vertebral metastases in patients with acute compression of the spinal cord.

\section{Patients and Methods}

Of 328 consecutive patients who had MRI of the spinal column at Newcastle General Hospital between September 1991 and May 1994, review of the case notes identified 115 with suspected compression of the spinal cord due to metastases. Of these, 100 fulfilled our criteria of MRI evidence of cord compression, a clinically documented neurological deficit, and histological confirmation of malignancy. There were 38 women and 62 men with a mean age of 63 years (18 to 83 ). Of the 15 patients who were excluded, seven had no MRI evidence of cord compression, five had no histological confirmation of malignancy, two had isolated involvement of the sacrum and one had incomplete MR images.

We used a 0.5 Tesla superconducting system scanner (MR MAX; GE Medical Systems, Milwaukee, Wisconsin) to obtain T1-weighted sagittal and axial views of the affected section of the vertebral column. The images were interpreted by a radiologist (SAW) who documented the following:

1) the level(s) of cord compression;

2) the site and extent of bony metastases in one of the four designated columns (1 anterior, 2 lateral and 1 posterior) shown in Figure 1;

3) the presence of a pathological fracture;

4) the pattern of fracture of a vertebral body, such as anterior or posterior wedging or central or uniform collapse;

5) the degree of collapse, as measured by the maximal loss of height as a percentage of the height of the nearest normal vertebra; and 


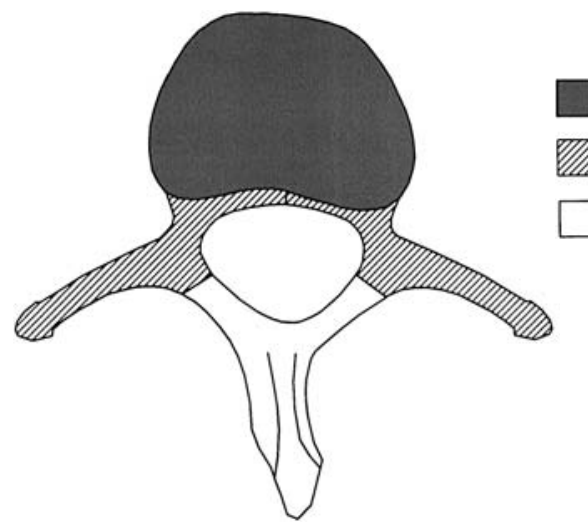

Fig. 1

Diagram showing the four designated spinal columns (1 anterior, 2 lateral and 1 posterior).

6) the site of cord compression in relation to quadrants corresponding to the four designated spinal columns. Cord compression was defined as an alteration in the outline and position of the spinal cord or cauda equina with an obliteration of the surrounding cerebrospinal fluid.

We studied the case notes to confirm the presence of a neurological deficit and a histological diagnosis of malignancy. Neurological deficit was documented as sensory loss, motor weakness, dysreflexia, or sphincter disturbance. All patients had either a tissue diagnosis of primary malignancy or a positive histological confirmation of vertebral metastases.

\section{Results}

Metastatic lesions were predominantly from primary tumours of the prostate, breast and lung. Table I gives the diagnoses of the patients. In cases in which the primary tumour was unknown, metastatic involvement was confirmed histologically by surgical biopsy.

Documentation of neurological deficit was available for all patients. There was muscle weakness in 91 , sensory loss
Table I. Primary tumour diagnosis and vertebral involvement for the 100 patients with acute compression of the spinal cord due to metastasis

\begin{tabular}{lccc}
\hline Tumour origin & $\begin{array}{c}\text { Number } \\
\text { of cases }\end{array}$ & $\begin{array}{l}\text { Number with } \\
\text { multiple-level } \\
\text { involvement }\end{array}$ & $\begin{array}{l}\text { Number of } \\
\text { compressing } \\
\text { vertebrae }\end{array}$ \\
\hline Prostate & 25 & 12 & 42 \\
Breast & 18 & 7 & 28 \\
Lung & 18 & 7 & 27 \\
Gastrointestinal tract & 7 & 5 & 14 \\
Kidney & 6 & 3 & 10 \\
Mesothelioma & 2 & 0 & 2 \\
Non-Hodgkin's lymphoma & 1 & 1 & 3 \\
Hodgkin's lymphoma & 1 & 1 & 2 \\
Parotid & 1 & 1 & 2 \\
Bladder & 1 & 0 & 1 \\
Melanoma & 1 & 0 & 1 \\
Larynx & 1 & 0 & 1 \\
Merkel's tumour & 1 & 1 & 2 \\
Ovary & 1 & 1 & 4 \\
Plasmacytoma & 1 & 0 & 1 \\
Myeloma & 1 & 1 & 2 \\
Unknown & 14 & 3 & 18 \\
Total & 100 & 43 & 160 \\
\hline
\end{tabular}

in 73, reflex abnormality in 67 and sphincter disturbance in 45. Seven patients had a single isolated deficit while $27 \mathrm{had}$ impairment of all four modalities. Isolated sphincter disturbance, a late manifestation of cord compression, was observed in only one patient.

Although bony involvement at multiple levels was present in 43 patients, only those sites with evidence of compression of the spinal cord were studied. One patient had compression at five levels, two at four, ten at three and 30 at two. These sites were usually adjacent to each other, but could be up to 13 vertebrae apart. A total of 160 vertebral levels was studied.

The cord was most commonly compressed in the thoracic spine (123 vertebrae, 77\%). The lumbar spine was the site of compression of the cord or cauda equina in 26 vertebrae $(16 \%)$. The cervical cord was affected in 11 vertebrae (7\%). Figure 2 shows the distribution of the compressive lesions. Of the major diagnostic groups, there was no significant predilection of a primary tumour for a particular region; the thoracic spine was the commonest in

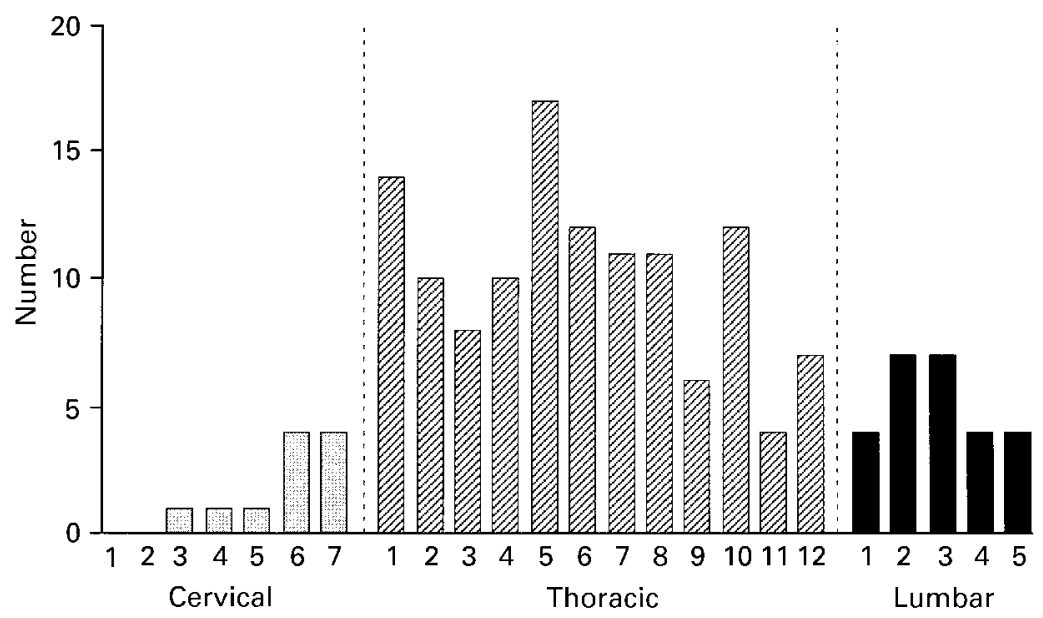

Fig. 2

The distribution of the levels of cord compression in the 100 patients.

VOL. 81-B, No. 5, SEPTEMBER 1999 


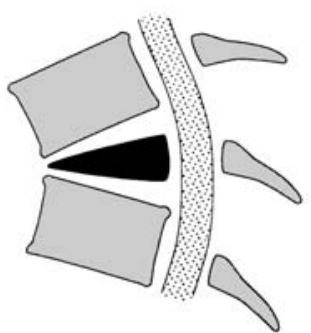

Anterior

19

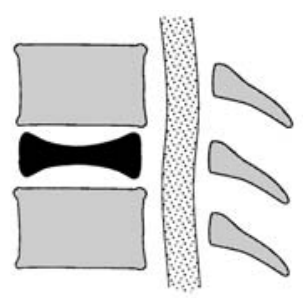

Central

40

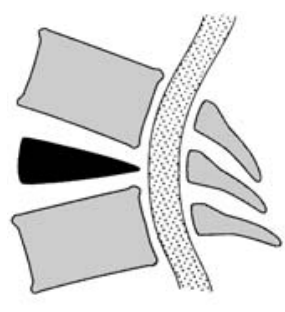

Posterior

4

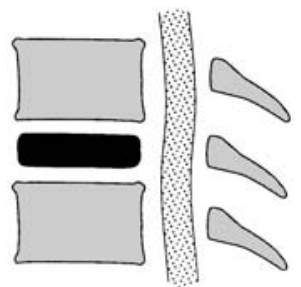

Uniform

18

Fig. 3

The patterns of vertebral collapse and the number of levels involved.

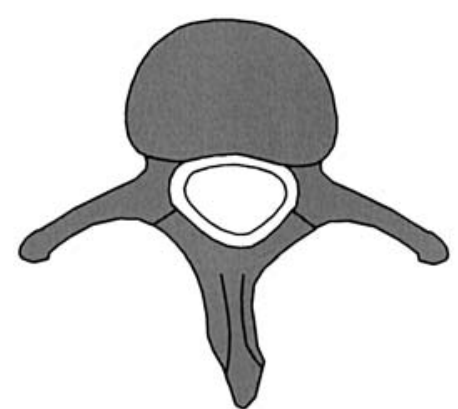

Four columns 83 levels $(52 \%)$

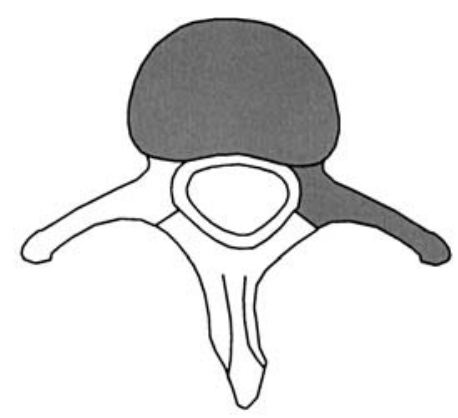

Two columns 22 levels $(14 \%)$

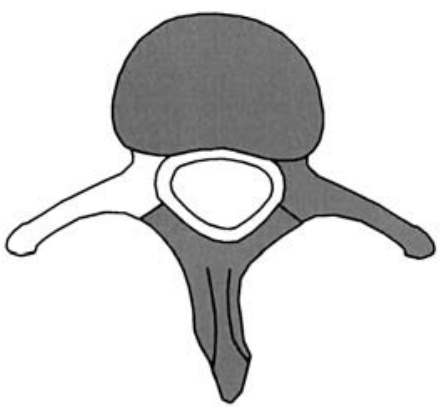

Three columns 49 levels $(30 \%)$

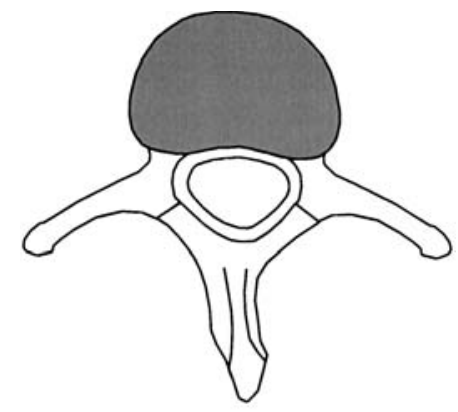

One column 6 levels $(4 \%)$

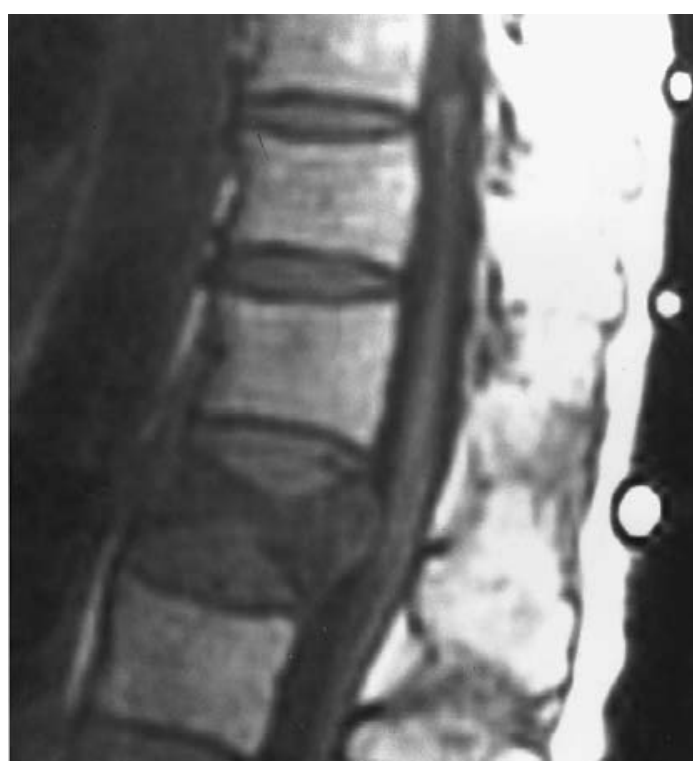

Fig. 4

T1-weighted sagittal view showing a pathological fracture with central collapse at T12 in a 64-year-old man with bronchial carcinoma. The cord is compressed from an anterior direction. all groups. There was no example of isolated extradural soft-tissue compression without bony involvement.

Pathological fracture of the vertebral body was present in 81 compressive levels (51\%) (Fig. 3). Of these, 55 (68\%) had a loss of height greater than $50 \%$. The most common pattern of collapse was central compression (Fig. 4). Vertebral bodies in the lower thoracic region were more likely to have pathological fractures. Prostatic lesions were least likely to give rise to collapse of the vertebral body.

Metastatic disease was present in the bodies of all vertebrae studied; most (155 vertebrae; $97 \%$ ) had more than $50 \%$ involvement as seen on MRI. The involvement of the four columns is summarised in Figure 5. Most vertebrae had four-column involvement and isolated single-column 


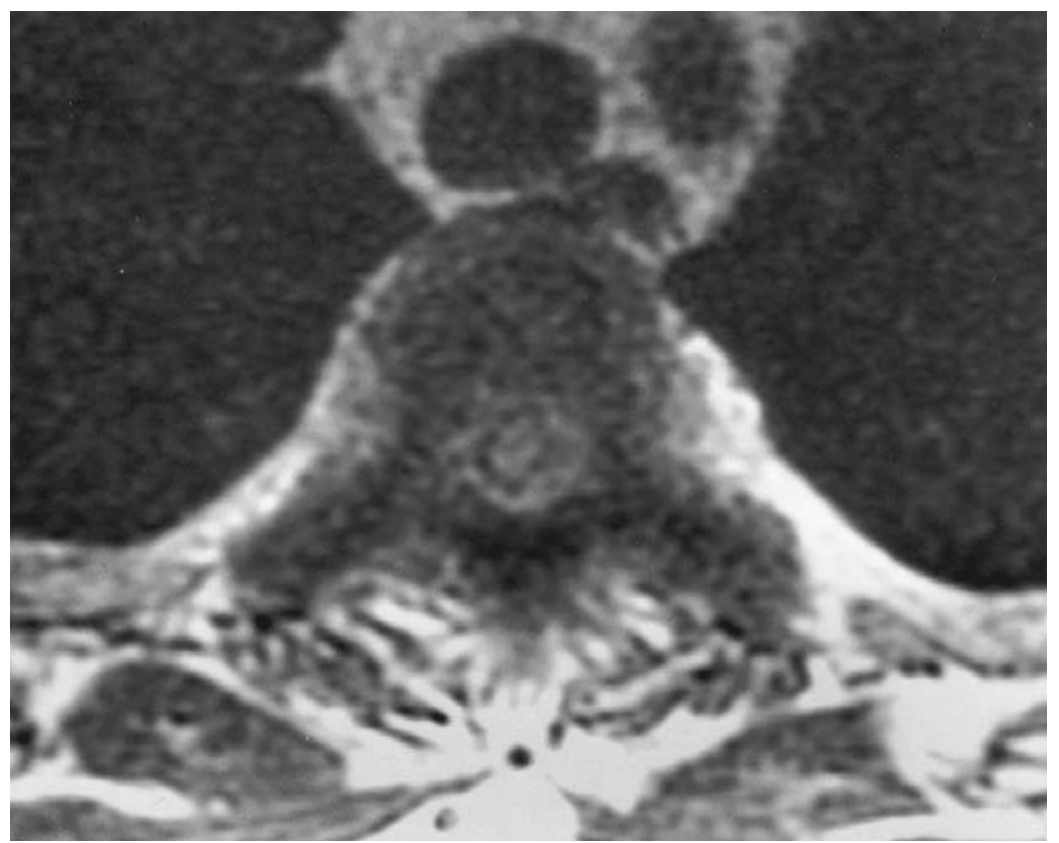

Fig. 6

T1-weighted axial view showing four-column metastatic bony involvement of T2 in a 62-year-old man with prostate carcinoma. There is also circumferential compression of the cord.

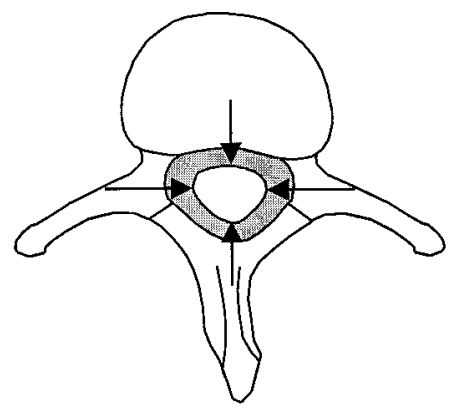

Circumferential 36 levels $(23 \%)$

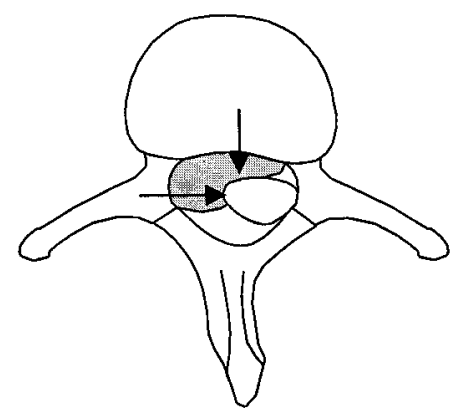

Two quadrants 34 levels $(21 \%)$

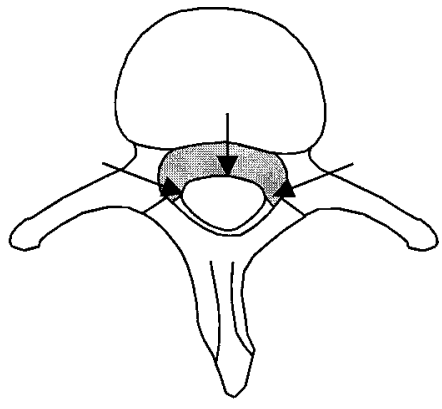

Three quadrants 75 levels $(47 \%)$

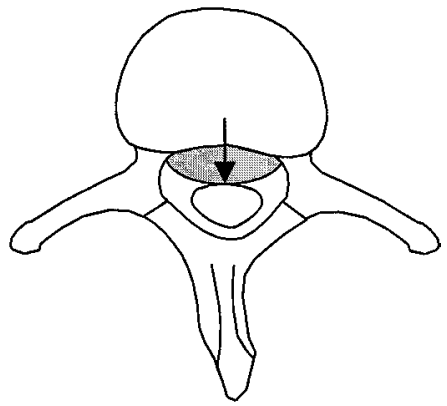

One quadrant 15 levels $(9 \%)$
Fig. 7

The site and direction of extradural soft-tissue compression of the cord and number of levels involved. Arrows indicate the most common directions of compression. involvement was present only in the anterior column. Coexisting anterior and posterior column disease was observed in 120 vertebrae (75\%) (Fig. 6).

The distribution of extradural soft-tissue compression is shown in Figure 7. Compression most commonly involved three quadrants and rarely affected an isolated single quadrant. Anterior compression (140 vertebrae, 87.5\%) was more common than posterior compression (84 vertebrae,
$52.5 \%$ ) and coexisting anterior and posterior cord compression was observed at 69 levels (43\%).

\section{Discussion}

It has been widely assumed that metastatic disease most often affects the vertebral body, and that compression of the cord is usually anterior. ${ }^{4}$ Involvement of the spinous pro- 
cesses and laminae, and compression from a posterior direction are often underestimated. In our series of patients presenting with acute compression of the spinal cord due to metastases, co-existing anterior and posterior structural involvement was common with bony involvement in $75 \%$ and soft-tissue compression of the cord in $43 \%$. Circumferential soft-tissue compression of the spinal cord was not unusual ( $22 \%$ of levels). Isolated anterior compression with metastatic lesions confined to the vertebral body was observed in only $3 \%$ of vertebrae.

Previous reports have suggested that there is often coexisting metastatic bone disease of the anterior and posterior columns. As early as 1924, Thompson and Keiller ${ }^{5}$ described extensive involvement of the posterior element in a case report. Studies using tomography and CT have also identified significant involvement of the posterior arch. ${ }^{6,7}$ More recently, MRI of a small series of patients has also shown this. 8,9

Most compressive lesions are at the level of the thoracic spine. ${ }^{6,10}$ The larger number of thoracic vertebrae is not sufficient explanation in itself. Other factors such as the unfavourable canal-to-cord ratio and the thoracic kyphus may explain why compression of the cord is more common at this level.

In our study, multiple-level compression is twice as common as that reported in other series. ${ }^{9-11}$ This may be due to the higher proportion of patients with carcinoma of the prostate.

The division of the vertebra into four columns allowed correlation with the four quadrants of the spinal canal. Clinically, such a classification is relevant in decompressive surgery in which, logically, decompression should be from the direction of maximal compression. The lateral column of the pedicle and transverse process is accessible from both anterior and posterior approaches, but the anterior and posterior columns are not easily reached from the opposite approach. This is an important consideration when compression is predominantly from one direction. A distinction is made between soft-tissue compression and bony metastatic involvement since the site of soft-tissue compression seems to bear little relationship to adjacent bony involvement. It is commonly thought that since metastatic disease usually affects the vertebral body, compression arises from an anterior direction. In our series, only nine vertebrae
(5.6\%) had isolated compression of the anterior cord of which five had lesions confined to the vertebral body. Isolated posterior compression is uncommon and was observed in only one patient who had extensive fourcolumn involvement.

A significant proportion of patients presenting with compression of the spinal cord due to metastases has coexisting anterior and posterior structural disease. Involvement of vertebral bodies is more common than posterior structural involvement in cord compression, suggesting a progression of the disease from anterior to posterior. Hence, the presence of posterior vertebral involvement in spinal metastases may predict imminent compression of the cord.

Our study has shown the value of MRI. The evaluation of both bony and soft-tissue involvement before planning surgical decompression is important.

No benefits in any form have been received or will be received from a commercial party related directly or indirectly to the subject of this article.

\section{References}

1. Li KC, Poon PY. Sensitivity and specificity of MRI in detecting malignant spinal cord compression and in distinguishing malignant from benign compression fractures of the vertebrae. Magn Reson Imaging 1988;6:547-56.

2. Smoker WR, Godersky JC, Knutzon RK, et al. The role of MR imaging in evaluating metastatic spinal disease. AJR-Am J Roentgenol 1987;149:1241-8.

3. Williams MP, Cherryman GR, Husband JE. Magnetic resonance imaging in suspected metastatic spinal cord compression. Clin Radiol 1989;40:286-90.

4. Harrington KD. Metastatic disease of the spine. J Bone Joint Surg [Am] 1986;68-A:1110-5.

5. Thompson JE, Keiller VH. Multiple skeletal metastases from cancer of the breast. Surg Gyn and Obs 1924;38:367-75.

6. Constans JP, DeDivitis E, Donzelli R, et al. Spinal metastases with neurological manifestations: review of 600 cases. J Neurosurgery 1983;59:111-8.

7. Manabe S, Tateishi A, Abe M, Ohno T. Surgical treatment of metastatic tumors of the spine. Spine 1989;14:41-7.

8. Lafforgue P, Bayle O, Massonnat $\mathbf{J}$, et al. MRI in osteoporotic and metastatic vertebral compressions: à propos of 60 cases. Ann Radiol Paris 1991;34:157-66.

9. Lien HH, Blomlie V, Heimdal K. Magnetic resonance imaging of malignant extradural tumors with acute spinal cord compression. Acta Radiol 1990;31:187-90.

10. Pigott KH, Baddeley H, Maher EJ. Pattern of disease in spinal cord compression on MRI scan and implications for treatment. Clin Oncol $R$ Coll Radiol 1994;6:7-10.

11. Gilbert RW, Kim J-H, Posner JB. Epidural spinal cord compression from metastatic tumor: diagnosis and treatment. Ann Neurol 1978; $3: 40-51$. 